\title{
SEMIPERFECT RINGS AND NAKAYAMA PERMUTATIONS
}

\author{
DINESH KHURANA \\ Centre for Advanced Study in Mathematics, Panjab University, Chandigarh-160014, India \\ e-mail:dkhurana@pu.ac.in
}

(Received 6 September, 2000; accepted 15 September 2001)

\begin{abstract}
We study the conditions which force a semiperfect ring to admit a Nakayama permutation of its basic idempotents. We also give a few necessary and sufficient conditions for a semiperfect ring $R$, which cogenerates every 2-generated right $R$-module, to be right pseudo-Frobenius.
\end{abstract}

2000 Mathematics Subject Classification. 16L30, 16L60.

0. Introduction. Throughout $R$ is an associative ring with identity and modules are unitary. The right and left annihilators of subset $X$ of a ring $R$ are denoted by $r_{R}(X)$ and $l_{R}(X)$ respectively. We write $J=J(R)$ for the Jacobson radical of a ring $R$ and $\operatorname{Soc}(M)$ for the socle of a module $M$. Right and left singular ideals of a ring $R$ will be denoted by $Z\left(R_{R}\right)$ and $Z\left({ }_{R} R\right)$ respectively. By $N \unlhd M$ we shall mean that $N$ is an essential submodule of a module $M$.

A ring $R$ is called right mininjective (right principally injective) if every $R$-homomorphism from a minimal (principal) right ideal of $R$ into $R$ is given by left multiplication by an element of $R$. Mininjective rings were introduced by Harada [7] who studied them in Artinian case. Recently Nicholson and Yousif [13] studied arbitrary mininjective rings. Principally injective rings have been studied in $[3,12,16,17]$. A ring $R$ is called right Kasch if $R$ contains a copy of each simple right $R$-module. An idempotent $e$ of a ring $R$ is called local if $e R e$ is a local ring; equivalently if $e J$ is the unique maximal submodule of $e R$. Nakayama [10] called a left and right Artinian ring $R$ with basic set of idempotents $e_{1}, \ldots, e_{n}$ quasi-Frobenius if there exists a permutation $\pi$ of $\{1, \ldots, n\}$ such that

$$
\operatorname{Soc}\left(R e_{\pi(i)}\right) \cong R e_{i} / J e_{i} \text { and } \operatorname{Soc}\left(e_{i} R\right) \cong e_{\pi(i)} R / e_{\pi(i)} J .
$$

Let $R$ be a semiperfect ring with basic set of idempotents $e_{1}, \ldots, e_{n}$. In this paper, following Nicholson and Yousif [12], we call a permutation $\sigma$ of $\{1, \ldots, n\}$ a Nakayama permutation if there exists a set $k_{1}, \ldots, k_{n}$ of elements of $R$ such that for each $i$

(1) $R k_{i} \subseteq R e_{\sigma(i)}$ and $k_{i} R \subseteq e_{i} R$

(2) $R k_{i} \cong R e_{i} / J e_{i}$ and $k_{i} R \cong e_{\sigma(i)} R / e_{\sigma(i)} J$.

In particular, $\left\{k_{1} R, \ldots, k_{n} R\right\}$ and $\left\{R k_{1}, \ldots, R k_{n}\right\}$ are complete irredundant sets of representatives of isomorphism classes of simple right and simple left $R$-modules respectively and so $R$ is left and right Kasch.

If a ring is right self injective and right cogenerator, it is called right pseudoFrobenius $(P F)$. Extending some well known results on PF and quasi-Frobenius (QF) rings, Nicholson and Yousif proved that a right minfull ring (that is a semiperfect right mininjective ring $R$ with $\operatorname{Soc}(e R) \neq 0$ for every local idempotent $e$ [13]) 
admits a Nakayama permutation of its basic idempotents and $\operatorname{Soc}(e R)$ is homogeneous for every local idempotent $e$. Moreover, its two socles are equal if every simple left ideal is a left annihilator [13, Theorem 3.7].

For a semiperfect ring $R$ admitting a Nakayama permutation of its basic idempotents, $\operatorname{Soc}(e R) \neq 0$ for every local idempotent $e$. Moreover, $\operatorname{Soc}(e R)$ is homogeneous for every local idempotent $e$ if and only if $R$ satisfies the following condition:

(*) For every local idempotents $e$ and $f$ of a ring $R$ if $e R$ and $f R$ contain isomorphic simple submodules then $e R \cong f R$.

Every right mininjective ring satisfies $(*)$ [13, Lemma 3.4]. A ring $R$ is called right minsymmetric [13] if $k R$ simple implies that $R k$ is simple, $k \in R$. A right mininjective ring is right minsymmetric [13, Theorem 1.14].

We prove that the mild condition of right minsymmetry ensures the existence of a Nakayama permutation of basic idempotents of a semiperfect ring $R$ satisfying $(*)$ for which $\operatorname{Soc}(e R) \neq 0$ for every local idempotent $e$. As even a commutative local ring with non-zero socle may not be minfull, this generalizes Nicholson and Yousif's result. For example,

$$
S=\left\{\left(\begin{array}{cc}
q & r \\
0 & q
\end{array}\right): q \in \mathbb{Q} \text { and } r \in \mathbb{R}\right\}
$$

is a commutative local ring which is not mininjective because $\operatorname{Soc}(S)=\left(\begin{array}{cc}0 & \mathbb{R} \\ 0 & 0\end{array}\right)$ is
not simple (see [13, Remark 1.4]).

In [13, Theorem 4.17] Nicholson and Yousif proved that a semiperfect right continuous ring with large right socle admits a Nakayama permutation of its basic idempotents. Also Yousif [18, Theorem 1] proved that a right CS ring $R$ such that the $R$-dual of every simple left $R$-module is simple, semiperfect and admits a Nakayama permutation of its basic idempotents. Improving upon these results, we prove that these classes of semiperfect rings are right minsymmetric and satisfy $(*)$.

Osofsky [15] proved that a ring $R$ is right PF if and only if it is semiperfect right self-injective with $\operatorname{Soc}\left(R_{R}\right) \unlhd R_{R}$. Recently, in their remarkable paper, Gómez Pardo and Guil Asensio [5, Corollary 2.8] proved that a right CS right cogenerator ring is right PF. We give some necessary and sufficient conditions for a semiperfect ring $R$, which cogenerates every 2 -generated right $R$-module, to be right PF. In particular, we prove that a semiperfect ring $R$ is right PF if and only if $J(R) \subseteq Z\left(R_{R}\right)$ and $R$ cogenerates every 2-generated right $R$-module. As every left CS right Kasch ring is semiperfect [6], this extends [14, Theorem 2.8] where it is proved that a left CS ring $R$ with $J(R) \subseteq Z\left(R_{R}\right)$ which cogenerates every 2-generated right $R$-module is right PF. In section 2 we study some relationships between right mininjective, right minsymmetric and left minannihilator rings (that is, rings for which every minimal left ideal is a left annihilator).

1. Nakayama permutations. A module $M$ is called a CS module if every submodule of $M$ is essential in a direct summand. A CS module is called continuous if it satisfies the following condition:

$\left(C_{2}\right)$. Every submodule of $M$ that is isomorphic to a direct summand of $M$ is itself a direct summand.

A CS module is called quasi-continuous if it satisfies the following condition: 
$\left(C_{3}\right)$. The sum of any two direct summands of $M$ whose intersection is zero is a direct summand.

Continuous modules are quasi-continuous [8]. A ring $R$ is said to be right CS if $R_{R}$ is a CS module. Right continuous and right quasi-continuous rings are defined similarily. For a detailed study of CS, quasi-continuous and continuous modules we refer the reader to [8].

We begin with the following result which will be used repeatedly in this paper.

Lemma 1.1. Let e be a local idempotent of a ring $R$. Then

(1) $J+(1-e) R$ is the unique maximal right ideal of $R$ containing $(1-e) R$;

(2) $J+R(1-e)$ is the unique maximal left ideal of $R$ containing $R(1-e)$;

(3) If $0 \neq K \subseteq R e$ is an annihilator left ideal then $\operatorname{Soc}\left(R_{R}\right) e \subseteq K$, where $e$ is a local idempotent.

Proof. (1) Consider the map $1 \rightarrow e+e J$ from $R \rightarrow e R / e J$. This is an epimorphism with kernel $J+(1-e) R$. As $e$ is local, $J+(1-e) R$ is a maximal right ideal. If $(1-e) R \subseteq I$, where $I$ is a maximal right ideal, then $(1-e) R+J \subseteq I+J=I$. This gives (1).

(2) This is proved similarly to (1).

(3) As $(1-e) R \subseteq r_{R}(K) \neq R$, by $(1), r_{R}(K) \subseteq J+(1-e) R$. Thus

$$
\begin{aligned}
K & =l_{R} r_{R}(K) \supseteq l_{R}((1-e) R+J)=l_{R}((1-e) R) \cap l_{R}(J) \\
& \supseteq \operatorname{Re} \cap \operatorname{Soc}\left(R_{R}\right)=\operatorname{Soc}\left(R_{R}\right) e .
\end{aligned}
$$

The following result generalizes the analogous result proved for right minfull rings in [13, Theorem 3.7].

TheOREM 1.2. Let $R$ be a semiperfect ring satisfying $(*)$ with $\operatorname{Soc}(e R) \neq 0$ for every local idempotent $e$. If $R$ is right minsymmetric, then $R$ admits a Nakayama permutation of its basic idempotents. Moreover, if every simple submodule $K \subseteq R e$, where $e$ is a local idempotent, is a left annihilator, then

(1) $\left\{\operatorname{Soc}\left(\operatorname{Re}_{1}\right), \ldots, \operatorname{Soc}\left(R e_{n}\right)\right\}$ is a complete irredundant set of representatives of simple left $R$-modules, where $e_{1}, \ldots, e_{n}$ is a basic set of idempotents of $R$;

(2) $\operatorname{Soc}\left(R_{R}\right)=\operatorname{Soc}\left({ }_{R} R\right)$;

(3) $R$ is right minfull.

Proof. Let $e_{1}, \ldots, e_{n}$ be a basic set of idempotents of $R$. As $\operatorname{Soc}\left(e_{i} R\right) \neq 0$ and $R$ satisfies $(*), \operatorname{Soc}\left(e_{i} R\right)$ is homogeneous for each $i$. Let $K_{i} \subseteq \operatorname{Soc}\left(e_{i} R\right)$ be simple. By $(*)$, $i \neq j$ implies $K_{i} \neq K_{j}$. Thus there exists a permutation $\sigma$ of $\{1, \ldots, n\}$ such that $e_{\sigma(i)} R / e_{\sigma(i)} J \stackrel{\phi_{i}}{\cong} K_{i}$ for each $i$. Let $\phi_{i}\left(\overline{e_{\sigma(i)}}\right)=k_{i}$. Then $K_{i}=k_{i} R$ and as $k_{i} e_{\sigma(i)}=k_{i}$, $R k_{i} \subseteq R e_{\sigma(i)}$. As $e_{i} k_{i}=k_{i} \neq 0, e_{i} R k_{i} \neq 0$ for each $i$. Also as $R$ is right minsymmetric and $k_{i} R=K_{i}$ is a simple, $R k_{i}$ is simple for each $i$. So, by [1, Exercise 27.9], $R k_{i} \cong R e_{i} / J e_{i}$. Thus $\sigma$ is a Nakayama permutation.

Now suppose that every minimal left ideal contained in $R e$, where $e^{2}=e$ is local, is a left annihilator. Let $K \subseteq R e$ be simple. By Lemma $1.1 \operatorname{Soc}\left(R_{R}\right) e \subseteq K$. As $R$ is right Kasch, $\operatorname{Soc}\left(R_{R}\right) e \neq 0$. So $K=\operatorname{Soc}\left(R_{R}\right) e$ implying that $K=\operatorname{Soc}(R e)=$ $\operatorname{Soc}\left(R_{R}\right) e$. Thus $\operatorname{Soc}\left(R_{R}\right)=\operatorname{Soc}\left({ }_{R} R\right)$ and $R k_{i}=\operatorname{Soc}\left(R e_{\sigma(i)}\right)$ for each $i$. This gives (1). Also as $\operatorname{Soc}\left(R_{R}\right) e$ is a simple left $R$-module for every local idempotent $e$, by [13, Theorem 3.2], $R$ is right mininjective and thus right minfull. 
Proposition 1.3. Let $R$ be a semiperfect right $C S$ ring with $\operatorname{Soc}\left(R_{R}\right) \subseteq \operatorname{Soc}\left({ }_{R} R\right)$. Then $R$ is right minsymmetric.

Proof. Let $k R$ be a minimal right ideal of $R$. As $R$ is right CS, there exists an idempotent $e$ of $R$ such that $k R \unlhd e R$. Clearly $e R$ is uniform and so $e$ is a local idempotent. As $k R \subseteq \operatorname{Soc}\left(R_{R}\right) \subseteq \operatorname{Soc}\left({ }_{R} R\right), R(1-e)+J \subseteq l_{R}(k) \neq R$. But $R(1-e)+J$ is a maximal left ideal by Lemma 1.1 , so $R(1-e)+J=l_{R}(k)$. Thus $R k \cong R / l_{R}(k)$ is a minimal left ideal.

REMARK 1.4. Proposition 1.3 also holds for right min-CS rings (that is rings whose minimal right ideals are essential in direct summands). The proof of Proposition 1.3 shows that if $R$ is any ring with $\operatorname{Soc}\left(R_{R}\right) \subseteq \operatorname{Soc}\left({ }_{R} R\right)$ then for any $0 \neq k \in \operatorname{Soc}(e R)$, where $e$ is a local idempotent, $R k$ is simple. This extends [13, Proposition 3.3(3)] where this result is proved for semiperfect right mininjective rings.

Lemma 1.5. ([9, Theorem 4]). In a quasi-continuous module closures of isomorphic submodules are isomorphic. In particular, a semiperfect right quasi-continuous ring satisfies $(*)$.

Proposition 1.6. Let $R$ be a semiperfect right CS ring with $\operatorname{Soc}\left(R_{R}\right) \unlhd R_{R}$. If $\operatorname{Soc}\left(R_{R}\right) \subseteq \operatorname{Soc}\left({ }_{R} R\right)$, then $R$ is a right minsymmetric ring satisfying $(*)$. In particular, $R$ admits a Nakayama permutation of its basic idempotents.

Proof. As $\operatorname{Soc}\left(R_{R}\right) \subseteq \operatorname{Soc}\left({ }_{R} R\right)$ and $\operatorname{Soc}\left(R_{R}\right) \unlhd R_{R}$, we have $\operatorname{Soc}\left({ }_{R} R\right) \unlhd R_{R}$. Thus $R$ is left Kasch (see [11, Lemma 3]). By [18, Lemma 1], $R$ satisfies the right $C_{2}$ condition and so $R$ is right continuous. Now the result follows from Proposition 1.3, Lemma 1.5 and Theorem 1.2.

The last sentence of the following result was proved in [13, Theorem 4.17].

COROLlary 1.7. A semiperfect right continuous ring $R$ with $\operatorname{Soc}\left(R_{R}\right) \unlhd R_{R}$ is right minsymmetric and satisfies $(*)$. In particular, $R$ admits a Nakayama permutation of its basic idempotents.

Proof. By [8, Proposition 3.5] $J=Z\left(R_{R}\right)$ and so $\operatorname{Soc}\left(R_{R}\right) \subseteq r_{R}\left(Z\left(R_{R}\right)\right)=$ $r_{R}(J)=\operatorname{Soc}\left({ }_{R} R\right)$. Now the result follows from Proposition 1.6.

Recently Gómez Pardo and Yousif [6] proved that a right CS left Kasch ring $R$ is semiperfect right continuous with $\operatorname{Soc}\left({ }_{R} R\right) \unlhd R_{R}$. Thus from Corollary 1.7 we have

COROLlary 1.8. A right CS left Kasch ring $R$ is semiperfect. Moreover, if $\operatorname{Soc}\left({ }_{R} R\right) \subseteq \operatorname{Soc}\left(R_{R}\right)$ then $R$ is right minsymmetric with $(*)$ such that $\operatorname{Soc}\left(R_{R}\right) \unlhd R_{R}$.

Corollary 1.9 ([18, Theorem 1]). A right CS ring $R$ such that the $R$-dual of every simple left $R$-module is simple is semiperfect right minsymmetric with (*) such that $\operatorname{Soc}\left(R_{R}\right) \unlhd R_{R}$.

Proof. By [13, Proposition 2.2] $R$ is left mininjective and so $\operatorname{Soc}\left({ }_{R} R\right) \subseteq \operatorname{Soc}\left(R_{R}\right)$. Thus the result follows from Corollary 1.8 . 
The following result was proved by Nicholson and Yousif in [12, Theorem 2.3] for right generalized pseudo-Frobenius (GPF) rings (that is semiperfect right principally injective rings with large right socle). We prove this result more generally for a semiperfect right principally injective ring $R$ with $\operatorname{Soc}(e R) \neq 0$ for every local idempotent $e$. The author does not know whether such rings are right GPF.

THEOREM 1.10. Let $e_{1}, \ldots, e_{n}$ be a basic set of idempotents in a semiperfect right principally injective ring with $\operatorname{Soc}\left(e_{i} R\right) \neq 0$ for each $i$. Then there exist elements $k_{1}, \ldots, k_{n}$ of $R$ and a permutation $\sigma$ of $\{1, \ldots, n\}$ such that the following hold for each $i$ :

(1) $R k_{i}=\operatorname{Soc}\left(R e_{\sigma(i)}\right) \cong R e_{i} / J e_{i}$ is simple and essential in $R e_{\sigma(i)}$. In particular, $R e$ is uniform for every local idempotent $e$;

(2) $\operatorname{Soc}\left(e_{i} R\right)$ is homogeneous with each simple submodule isomorphic to $e_{\sigma(i)} R / e_{\sigma(i)} J$;

(3) $\left\{R k_{1}, \ldots, R k_{n}\right\}$ is a complete irredundant set of representatives of isomorphism classes of simple left $R$-modules;

(4) $\left\{k_{1} R, \ldots, k_{n} R\right\}$ is a complete irredundant set of representatives of isomorphism classes of simple right $R$-modules;

(5) $\operatorname{Soc}\left(R_{R}\right)=\operatorname{Soc}\left({ }_{R} R\right)=\oplus_{i=1}^{n} R k_{i} R$ is essential in ${ }_{R} R$ and is finitely generated as a left $R$-module;

(6) $R k_{i} R$ is the homogeneous component of $\operatorname{Soc}\left(R_{R}\right)$ containing $k_{i} R$ and $R k_{i} R$ is the homogeneous component of $\operatorname{Soc}\left({ }_{R} R\right)$ containing $R k_{i}$.

Proof. Let $k_{1}, \ldots, k_{n}$ and $\sigma$ be as in Theorem 1.2. Then (2), (3) and (4) follow from Theorem 1.2.

(1) Let $0 \neq b \in R e_{\sigma(i)}$. As $\left(1-e_{\sigma(i)}\right) R \subseteq r_{R}(b)$, by Lemma 1.1, $r_{R}(b) \subseteq$ $\left(1-e_{\sigma(i)}\right) R+J \subseteq r_{R}\left(k_{i}\right)$ because $R k_{i} \subseteq R e_{\sigma(i)}$ is simple. Thus, by [12, Lemma 1.1], $R k_{i} \subseteq R b$.

(6) By [13, Theorem 1.14(3)] the homogeneous component of $\operatorname{Soc}\left(R_{R}\right)$ containing $k_{i} R$ is $R k_{i} R$. Let $S_{i}$ be the homogeneous component of $\operatorname{Soc}\left({ }_{R} R\right)$ containing $R k_{i}$. Clearly $R k_{i} R \subseteq S_{i}$. Let $f_{1}, \ldots, f_{m}$ be a complete orthogonal set of primitive idempotents of $R$. By (1) above, $S_{i}=\oplus\left\{\operatorname{Soc}\left(R f_{j}\right): R f_{j} \cong R e_{\sigma(i)}\right\}$. Now if $R f_{j} \cong R e_{\sigma(i)}$ then there exists $b \in R$ such that $\operatorname{Soc}\left(R f_{j}\right)=\operatorname{Soc}\left(R e_{\sigma(i)}\right) b=R k_{i} b$. Thus $S_{i} \subseteq R k_{i} R$.

(5) Follows from Theorem 1.2(2), and (1) and (6) above.

COROLlary 1.11. Let $R$ be a semiperfect right principally injective ring with $\operatorname{Soc}(e R) \neq 0$ for every local idempotent e. If $S=\operatorname{Soc}\left(R_{R}\right)=\operatorname{Soc}\left({ }_{R} R\right)$ then

(1) $Z\left(R_{R}\right)=J=Z\left({ }_{R} R\right)$;

(2) $l_{R}(S)=J=r_{R}(S)$;

(3) $l_{R}(J)=S=r_{R}(J)$.

Proof. Using Theorem 1.10, the proof follows the same lines as that of $[\mathbf{1 2}$, Corollary 2.2].

There exists a left and right Artinian ring $R$ such that every left ideal of $R$ is a left annihilator, but $R$ is not quasi-Frobenius [2, page 70]. Clearly $R$ is right principally injective with large right socle. But $R$ is not left mininjective as right Artinian, left and right mininjective rings are quasi-Frobenius [13, Corollary 4.8].

The next result gives several characterizations of right PF rings. Since left CS right Kasch rings are semiperfect [6], the implication '(4) $\Rightarrow$ (1)' extends

[14, Theorem 2.8]. 
THEOREM 1.12. Let $R$ be a semiperfect ring which cogenerates every 2-generated right $R$-module. Then the following are equivalent:

(1) $R$ is right $P F$;

(2) $\operatorname{Soc}\left(R_{R}\right) \subseteq \operatorname{Soc}\left({ }_{R} R\right)$;

(3) $\operatorname{Soc}(R e) \neq 0$ for every local idempotent e of $R$;

(4) $J(R) \subseteq Z\left(R_{R}\right)$.

Proof. As $R$ cogenerates every cyclic right $R$-module, every right ideal is a right annihilator (see for instance [1, Lemma 25.2]) and so $R$ is left principally injective. By [12, Theorem 1.14] $\operatorname{Soc}\left({ }_{R} R\right) \subseteq \operatorname{Soc}\left(R_{R}\right)$. Also as $R$ is right $\operatorname{Kasch} \operatorname{Soc}\left(R_{R}\right) e \neq 0$ for every local idempotent $e$ of $R$.

(1) $\Rightarrow(2)$ This is well-known.

(2) $\Rightarrow$ (3) We have $\operatorname{Soc}\left(R_{R}\right)=\operatorname{Soc}\left({ }_{R} R\right)$. Thus $\operatorname{Soc}(\operatorname{Re})=\operatorname{Soc}\left({ }_{R} R\right) e=\operatorname{Soc}\left(R_{R}\right) e$ $\neq 0$ for every local idempotent $e$ of $R$.

(3) $\Rightarrow$ (4) This follows from Corollary 1.11.

(4) $\Rightarrow$ (1) As $J(R) \subseteq Z\left(R_{R}\right), \operatorname{Soc}\left(R_{R}\right) \subseteq r_{R}\left(Z\left(R_{R}\right)\right) \subseteq r_{R}(J(R))=\operatorname{Soc}\left({ }_{R} R\right)$ and so $\operatorname{Soc}\left(R_{R}\right)=\operatorname{Soc}\left({ }_{R} R\right)$. For every local idempotent $e$ of $R, \operatorname{Soc}(R e)=\operatorname{Soc}\left({ }_{R} R\right) e=$ $\operatorname{Soc}\left(R_{R}\right) e \neq 0$ and so, by the proof of Theorem 1.10(1), $e R$ is uniform. Thus $E(e R)$, the injective hull of $e R$, is also uniform for every local idempotent $e$.

Fix a local idempotent $e$ in $R$. We show that $e R=E(e R)$. Suppose, on the contrary, $a \in E(e R) \backslash e R$. As $e R+a R$ is uniform with non-zero socle (as $\operatorname{Soc}(e R) \neq 0$ ), it is finitely co-generated right $R$-module [1, Proposition 10.7]. So, by hypothesis, there exists an embedding $e R+a R \rightarrow R^{n}$ for some natural number $n$. As $e R+a R$ is uniform, we have an embedding $\sigma: e R+a R \rightarrow f R$ for some local idempotent $f$ in $R$ (see for example [14, Lemma 2.6]). As $a \notin e R, \sigma(e R)$ is a proper submodule of $f R$ and so $\sigma(e R) \subseteq f J \subseteq Z\left(R_{R}\right)$. But as $r_{R}(\sigma(e))=(1-e) R$, this is a contradiction. Thus $e R=E(e R)$ is injective for every local idempotent $e$ of $R$ and so $R$ is right selfinjective. As $R$ is right Kasch, $R$ is right PF [1, Proposition 18.15].

It is not known whether a right perfect right self-injective ring is right PF. For a detailed account of this problem we refer the reader to [4].

Proposition 1.13. Let $R$ be a semiperfect ring satisfying $(*)$ with $\operatorname{Soc}(e R) \neq 0$ for every local idempotent e. If $\operatorname{Soc}\left(R_{R}\right)$ is finitely generated then for any projective right $R$-modules $P$ and $Q$, Soc $(P) \cong \operatorname{Soc}(Q)$ implies that $P \cong Q$.

Proof. Let $e_{1}, \ldots, e_{n}$ be a basic set of idempotents of $R$. By [1, Theorem 27.11] for each $i$ there exist sets $I_{i}$ and $J_{i}$ such that

$$
P \cong \bigoplus_{i=1}^{n}\left(e_{i} R\right)^{\left(I_{i}\right)} \text { and } Q \cong \oplus_{i=1}^{n}\left(e_{i} R\right)^{\left(J_{i}\right)} .
$$

Now $\operatorname{Soc}(P) \cong \operatorname{Soc}(Q)$ yields $\oplus_{i=1}^{n} \operatorname{Soc}\left(e_{i} R\right)^{\left(I_{i}\right)} \cong \oplus_{i=1}^{n} \operatorname{Soc}\left(e_{i} R\right)^{\left(J_{i}\right)}$. By hypothesis there exists a set $\left\{S_{1}, \ldots, S_{n}\right\}$ of mutually non-isomorphic simple right $R$-modules such that $\operatorname{Soc}\left(e_{i} R\right) \cong S_{i}^{k_{i}}$ for some natural number $k_{i}(1 \leq i \leq n)$. Let $|S|$ denote the cardinality of set $S$. Then $\oplus_{i=1}^{n} \operatorname{Soc}\left(e_{i} R\right)^{\left(I_{i}\right)} \cong \oplus_{i=1}^{n} \operatorname{Soc}\left(e_{i} R\right)^{\left(J_{i}\right)}$ implies that $\oplus_{i=1}^{n}\left(S_{i}^{k_{i}}\right)^{\left(I_{i}\right)} \cong \oplus_{i=1}^{n}\left(S_{i}^{k_{i}}\right)^{\left(J_{i}\right)}$ which, in turn, gives $\left(S_{i}^{k_{i}}\right)^{\left(I_{i}\right)} \cong\left(S_{i}^{k_{i}}\right)^{\left(J_{i}\right)}$ for each $i$. Thus, $\left|k_{i} \times I_{i}\right|=\left|k_{i} \times J_{i}\right|$ and so $\left|I_{i}\right|=\left|J_{i}\right|$, proving that $P \cong Q$.

REMARK 1.14. Let $R$ be a semiperfect ring with basic set of idempotents $\left\{e_{1}, \ldots, e_{n}\right\}$. Let there exist a set $\left\{S_{1}, \ldots, S_{n}\right\}$ of mutually non-isomorphic simple right 
$R$-modules, such that $\operatorname{Soc}\left(e_{i} R\right) \cong S_{i}^{k_{i}}$ for some natural number $k_{i}(1 \leq i \leq n)$. Then the proof of Proposition 1.13 shows that for any projective right $R$-modules $P$ and $Q, \operatorname{Soc}(P) \cong \operatorname{Soc}(Q)$ implies that $P \cong Q$.

The following result slightly strengthens [13, Theorem 3.16].

COROLlary 1.15. Let $R$ be a left minfull ring with $r_{R} l_{R}(K)=K$ for every simple right ideal $K \subseteq e R$, where $e^{2}=e$ is local. Then for any projective right $R$-modules $P$ and $Q$, $\operatorname{Soc}(P) \cong \operatorname{Soc}(Q)$ implies that $P \cong Q$.

Proof. Let $e_{1}, \ldots, e_{n}$ be a basic set of idempotents of $R$. By the proof of Theorem $1.2(1)\left\{\operatorname{Soc}\left(e_{1} R\right), \ldots, \operatorname{Soc}\left(e_{n} R\right)\right\}$ is a complete irredundant set of representatives of simple right $R$-modules. Thus the result follows from Remark 1.14.

As every principal right ideal of a left principally injective ring is a right annihilator, the following is a consequence of Corollary 1.15 .

COROllary 1.16. Let $R$ be a semiperfect left principally injective ring with $\operatorname{Soc}(R e) \neq 0$ for every local idempotent e of $R$. Then for any projective right $R$-modules $P$ and $Q$, $\operatorname{Soc}(P) \cong \operatorname{Soc}(Q)$ implies that $P \cong Q$.

2. Mininjective rings. As mentioned above every right mininjective ring is right minsymmetric. Also a left minannihilator ring is right mininjective if $\operatorname{Soc}\left(R_{R}\right) \subseteq$ $\operatorname{Soc}\left({ }_{R} R\right)$ [13, Proposition 2.4] or $\operatorname{Soc}\left({ }_{R} R\right) \unlhd{ }_{R} R$ [13, Corollary 2.5]. Thus right mininjective rings are closely related to right minsymmetric and left minannihilator rings. In this section we study relationships between these conditions.

Consider the following condition on a ring $R$ :

(**) Every minimal right ideal of $R$ is isomorphic to eR/eJ for some local idempotent $e$ of $R$.

Clearly every semiperfect ring satisfies (**). A ring $R$ satisfies (**) if and only if for every minimal right ideal $K$ of $R$ there exists a local idempotent $e$ of $R$ such that $K e \neq 0$ (see [1, Exercise 27.9]).

Lemma 2.1. ([13, Lemma 3.1]). Let $R$ be a ring satisfying (**). Then $R$ is right mininjective if and only if for every local idempotent e of $R$ either $\operatorname{Soc}\left(R_{R}\right)$ e is simple or zero.

Proposition 2.2. Let $R$ be a ring satisfying (**). Then

(1) If for every local idempotent $e$ of $R$ there exists a minimal left ideal in $\operatorname{Soc}(R e)$ which is a left annihilator then $R$ is right mininjective;

(2) If for every local idempotent e either $\operatorname{Soc}(R e)=0$ or every minimal left ideal contained in Re is a left annihilator, then the following are equivalent:

(a) $R$ is right mininjective;

(b) $R$ is right minsymmetric;

(c) $\operatorname{Soc}\left(R_{R}\right) \subseteq \operatorname{Soc}\left({ }_{R} R\right)$.

Proof. (1) Let $e$ be a local idempotent of $R$ and $K$ be a minimal left ideal in $R e$ such that $l_{R} r_{R}(K)=K$. By Lemma $1.1 \operatorname{Soc}\left(R_{R}\right) e \subseteq K$. So either $\operatorname{Soc}\left(R_{R}\right) e=0$ or $\operatorname{Soc}\left(R_{R}\right) e=K$ is simple. Thus, by Lemma $2.1, R$ is right mininjective. 
(2) (a) $\Rightarrow$ (b) follows from [13, Theorem 1.14] and (b) $\Rightarrow$ (c) is clear.

(c) $\Rightarrow$ (a) Let $e$ be a local idempotent of $R$. In view of Lemma 2.1 we have to show that $\operatorname{Soc}\left(R_{R}\right) e$ is either zero or simple. If $\operatorname{Soc}(R e)=0$ then $\operatorname{Soc}\left(R_{R}\right) e \subseteq \operatorname{Soc}\left({ }_{R} R\right) e=$ $\operatorname{Soc}(R e)=0$. Now suppose that $\operatorname{Soc}(R e) \neq 0$ and let $K \subseteq R e$ be simple. By Lemma 1.1 $\operatorname{Soc}\left(R_{R}\right) e \subseteq K$. Thus either $\operatorname{Soc}\left(R_{R}\right) e=0$ or $\operatorname{Soc}\left(R_{R}\right) e=K$ is simple.

Lemma 2.3. Let $R$ be a right Kasch ring with every minimal left ideal $K \subseteq \operatorname{Re} a$ left annihilator, where $e$ is a local idempotent. Then either $\operatorname{Soc}(\operatorname{Re})=0$ or $\operatorname{Soc}(R e)=\operatorname{Soc}\left(R_{R}\right)$ e is a simple left $R$-module.

Proof. Let $\operatorname{Soc}(R e) \neq 0$ and $K \subseteq$ Re be simple. By Lemma $1.1 \operatorname{Soc}\left(R_{R}\right) e \subseteq K$. As $R$ is right Kasch, $\operatorname{Soc}\left(R_{R}\right) e \neq 0$ and so $K=\operatorname{Soc}\left(R_{R}\right) e$. As $K$ is an arbitrary simple submodule of $R e, \operatorname{Soc}(R e)=\operatorname{Soc}\left(R_{R}\right) e$ is a simple left $R$-module.

The equivalence of following conditions was observed by Nicholson and Yousif in [13, Proposition 3.3 (4)] for semiperfect right mininjective right Kasch rings. We prove that these equivalences also hold for non-semiperfect rings.

Proposition 2.4. Let e be a local idempotent in a right mininjective right Kasch ring $R$. Then the following are equivalent:

(1) $l_{R} r_{R}(K)=K$ for every minimal left ideal $K \subseteq R e$;

(2) $\operatorname{Soc}(R e)=\operatorname{Soc}\left(R_{R}\right) e$;

(3) $\operatorname{Soc}(R e)$ is simple.

Proof. As $R$ is right Kasch, $\operatorname{Soc}\left(R_{R}\right) e \neq 0$. Also by [13, Theorem 1.14] $\operatorname{Soc}\left(R_{R}\right) \subseteq \operatorname{Soc}\left({ }_{R} R\right)$.

(1) $\Rightarrow$ (2) As $0 \neq \operatorname{Soc}\left(R_{R}\right) e \subseteq \operatorname{Soc}\left({ }_{R} R\right) e=\operatorname{Soc}(R e)$, by Lemma 2.3 we find $\operatorname{Soc}(R e)=\operatorname{Soc}\left(R_{R}\right) e$.

(2) $\Rightarrow$ (3) As $R$ is right mininjective, by [13, Lemma 3.1], $\operatorname{Soc}\left(R_{R}\right) e$ is zero or simple. But as $\operatorname{Soc}\left(R_{R}\right) e \neq 0, \operatorname{Soc}(R e)=\operatorname{Soc}\left(R_{R}\right) e$ is simple.

(3) $\Rightarrow$ (1) Let $K \subseteq R e$ be simple. Then $0 \neq \operatorname{Soc}\left(R_{R}\right) e \subseteq \operatorname{Soc}\left({ }_{R} R\right) e=$ $\operatorname{Soc}(R e)=K$. This gives $K=\operatorname{Soc}\left(R_{R}\right) e \subseteq l_{R}(J) e \cong H o m\left(\frac{e R}{e J}, R\right)$. As $R$ is right mininjective, the $R$-dual of every simple right $R$-module is either zero or a simple left $R$-module [13, Proposition 2.2]. Thus $K=l_{R}(J) e=l_{R}(J) \cap R e=l_{R}(J) \cap l_{R}((1-e) R)=$ $l_{R}(J+(1-e) R)$.

ACKnOwLEDGEments. The author would like to thank the referee for some useful suggestions.

\section{REFERENCES}

1. F. W. Anderson and Kent R. Fuller, Rings and categories of modules (Springer-Verlag, GTM No 13, 1974).

2. J.-E. Björk, Rings satisfying certain chain conditions, J. Reine. Angew. Math. 245 (1970), 63-73

3. V. Camillo, Commutative rings whose principal ideals are annihilators, Portugal. Math. 46 (1989), 33-37.

4. Dinesh Khurana, Is a right perfect right self-injective ring right PF?, Vietnam J. Math. 28 (2000), 321-328. 
5. J. L. Gómez Pardo and P. A. Guil Asensio, Rings with finite essential socle, Proc. Amer. Math. Soc. 125 (1997), 971-977.

6. J. L. Gómez Pardo and M. F. Yousif. Semiperfect min-CS rings, Glasgow Math. J. 41 (1999), 231-238.

7. M. Harada, Self mini-injective rings, Osaka J. Math. 19 (1982), 587-597.

8. S. Mohamed and B. Müller, Continuous and discrete modules, London Math. Soc. Lecture Note Series No 147 (Cambridge University Press, 1990).

9. B. J. Müller and S. T. Rizvi, On injective and quasi-continuous modules, J. Pure Appl. Alg. 28 (1983), 197-210.

10. T. Nakayama, On Frobeniusean algebras, Ann. of Math. 42 (1941), 1-21.

11. W. K. Nicholson and M. F. Yousif, Continuous rings with chain conditions, J. Pure. Appl. Alg. 97 (1994), 325-332. $77-93$.

12. W. K. Nicholson and M. F. Yousif, Principally injective rings, J. Algebra 174 (1995),

13. W. K. Nicholson and M. F. Yousif, Mininjective rings, J. Algebra 187 (1997), 548578.

14. W. K. Nicholson and M. F. Yousif, Annihilators and the CS-condition, Glasgow Math. J. 40 (1998), 213-222.

15. B. L. Osofsky, A generalization of quasi-Frobenius rings, J. Algebra 4 (1966), 373387. 212.

16. E. A. Rutter, Rings with principal extension property, Comm. Algebra 3 (1975), 203-

17. W. Xue, A note on principally injective rings, Comm. Algebra 26 (1998), 4187-4190.

18. M. F. Yousif, CS rings and Nakayama permutations, Comm. Algebra 25 (1997), $3787-3795$. 